\title{
Die Lunge und ihre Abwehrmechanismen
}

\author{
U. Costabel $\left.{ }^{1}\right)$, C. Kroegel ${ }^{2}$ ) \\ 1) Abteilung Pneumologie/Allergologie der Ruhrland-Klinik Essen \\ ${ }^{2}$ ) Abteilung Pneumologie der Medizinischen Universitätsklinik \\ Freiburg im Breisgau
}

\section{Zusammenfassung}

Die Lunge verfügt über vielfältige Abwehrmechanismen gegen eingeatmete Schadstoffe. Im trachebronchialen Bereich erfüllt das intakte Epithel der Bronchialschleimhaut als undurchlässige Barriere bereits eine wichtige Schutzfunktion. Mukoziliäre und tussive Clearance befördern inhalierte Partikel nach außen. Im Bronchialsekret gelöste Komponenten der immunologischen Abwehr wie sekretorisches IgA, Lysozym, Interferon und Komplementfaktoren stellen wirksame Verteidigungsprinzipien gegen eindringende infektiöse Erreger dar. Im alveolären Bereich nimmt der Alvolarmakrophage eine zentrale Position ein. Neben der Phagozytosefunktion gibt es kaum eine immunologische Reaktion, an der er nicht direkt oder indirekt (über Monokine, Enzyme, Entzündungsmediatoren) beteiligt ist. Weitere Immunzellen in den Alveden sind neutrophile und eosinophile Granulozyten, Mastzellen, sowie B- und T-Lymphozyten als zelluläre Komponenten der spezifischen Immunität. Während sich B-Lymphozyten nach Antigenkontakt zu antikörperbildenden Plasmazellen transformieren, erfüllen die Subpopulationen der T-Lymphozyten immunregulatorische Funktionen. T4-Helferzellen wirken stimulierend und T8-Suppressorzellen inhibierend auf die B-Zellreaktion und andere Immunreaktionen. Der T4-positive Lymphozyt stellt die Effektorzelle der Immunreaktion vom verzögerten Typ (Typ IV) dar. Der T8-positive Lymphozyt kann als zytotoxische Effektorzelle virusbefallene Zellen, Tumorzellen und Transplantatzellen erkennen und vernichten. Die HIV-Infektion mit Befall und Zerstörung der T4-Zellen trifft das Immunsystem an zentraler Stelle und führt zum weitgehenden Ausfall der immunologischen Abwehr.

\section{Einleitung}

Die Lunge verfügt über ein besonders effektives und gut zusammenwirkendes Abwehrund Reinigungssystem, das sie als das größte Kontaktorgan unseres Körpers zur atmosphärischen Umwelt auch benötigt. Bei einer Oberfläche der Alveolen von etwa $100 \mathrm{qm}$ und einer täglichen Durchströmung mit 15.000 bis 20.0001 Luft müssen die eingeatmeten Schadstoffe (Stäube, Bakterien, Viren u. a.) wieder eliminiert werden. Tabelle 1 zeigt, daß man die Abwehrmechanismen nach ihrer Lokalisation einem trachebronchialen Kompartiment zuordnen kann, welches aus dem intakten Epithel der Bronchialschleimhaut, der tussiven Clearence, der mukoziliären Clearene und dem Bronchialsekret mit Inhaltstoffen besteht. Im alveolären Kompartiment spielen der Surfactant und vor allem die Alveolarmakrophagen neben weiteren Immunzellen eine bedeutende Rolle. Ein besonderes System stellt die immunologische Abwehr dar (31), die in beiden obenge- 
Tabelle 1. Abwehrmechanismen der Lunge.

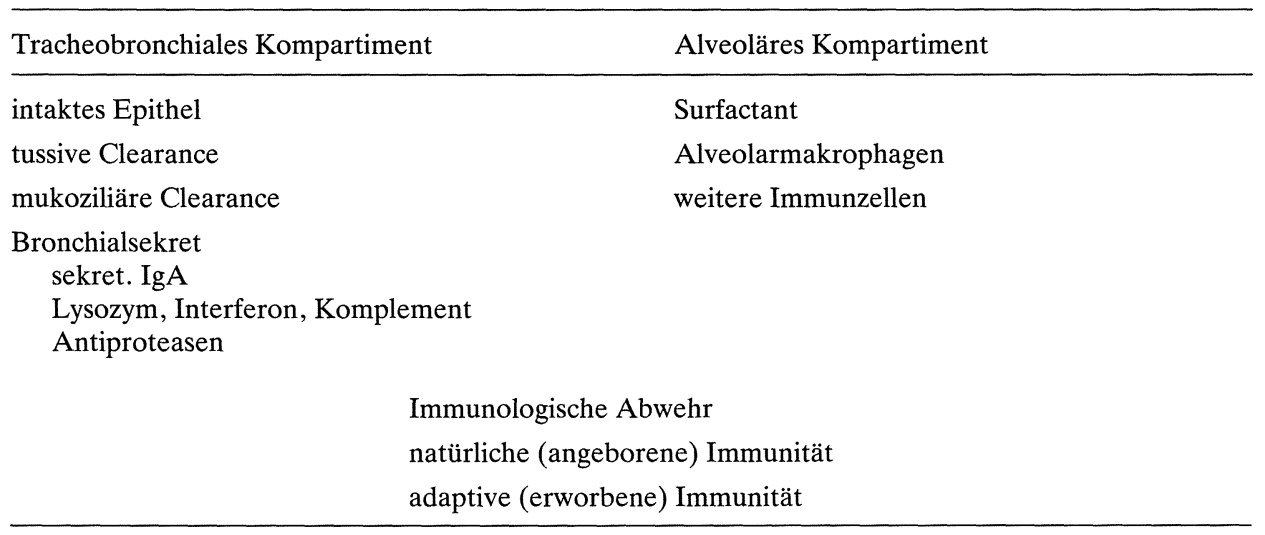

nannten Kompartimenten zum Tragen kommt und die man in die natürliche (angeborene) Immunität, früher auch unspezifische Immunität genannt, und in die adaptive (erworbene) oder spezifische Immunität einteilen kann.

\section{Trachebronchiales Kompartiment}

Die Hauptfunktion des Epithels ist es zunächst, eine intakte, undurchlässige Barriere gegen alle möglichen Arten von Schadstoffen zu bilden. Eine besondere Bedeutung kommt dabei den sogenannten »Tight junctions « zu, bei denen es sich um enge Verzahnungen zweier benachbarter Zellen handelt und die das Epithel besonders undurchlässig gestalten. Morphologisch verfügt das Bronchialepithel über drei verschiedene Zellarten, denen Spezialfunktionen zukommen: zilientragende Flimmerepithelien, schleimbildenden Becherzellen und Basalzellen, aus denen sich die beiden erstgenannten Zellformen regenerieren. Von den in der Submukosa gelegenen seromukösen Drüsen wird die Hauptmasse des Schleims des menschlichen Bronchialsystems gebildet. Ein optimal beschaffenes Bronchialsekret (nicht zu dünn, nicht zu zäh) in Verbindung mit einer geordneten Zilientätigkeit des Flimmerepithels trägt zur mukoziliären Clearance inhalierter Schadstoffe bei. Das Bronchialsekret besteht aus 2 Phasen, einer oberen visköseren Gelschicht, in der die Partikel transportiert werden, und einer unteren, mehr wässrigen Solschicht, in der sich die Zilien mit einer Frequenz von $12 \mathrm{~Hz}$ bewegen (24). Der rollende Teppich der mukoziliären Clearance befördert so die Mehrzahl der inhalierten Partikel in den Rachen. Falls diese Flimmerepithelien, z. B. durch Zigarettenrauch, gelähmt sind, kann das Sekret nicht in ausreichender Menge oralwärts befördert werden. Als Ersatzmechanismus tritt dann die tussive Clearance verstärkt in Kraft. Jeder Hustenstoß bezweckt den Weitertransport des Sekrets über Flimmerepithelstrecken, die nicht mehr ausreichend funktionieren und auf denen der Schleim liegenzubleiben droht.

Um zu verhindern, daß im Bronchialsekret deponierte Krankheitserreger wie Viren oder Bakterien sich dort ungehemmt ausbreiten und dann doch die intakte Epithelbarriere überwinden, sind im Bronchialsekret sehr wirksame immunologische Verteidigungsprinzipien in Form löslicher Substanzen vorhanden. Da ist einmal das sekretorische 
IgA als Teilkomponente der adaptiven Immunität, gebildet von Plasmazellen der Submukosa. Ferner sind es lösliche Komponenten der natürlichen Immunität wie Lysozym, Interferone, Komplementfaktoren und auch Antiproteasen, die hier ihre Schutzfunktion ausüben (1).

Das sekretorische IgA ist im Gegensatz zum im Serum vorkommenden IgA ein Dimer, das heißt, zwei IgA-Monomere sind zu einer größeren Komponente mit einem Molekulargewicht von 385.000 verknüpft (21). Zusätzlich verbindet diese beiden Monomere eine sekretorische Komponente, die von Bronchialepithelien gebildet wird. Diese schützt das sekretorische IgA vor Proteolyse. Durch Entzündungszellen freigesetzte Proteasen könnten ungeschützte Immunglobuline rasch inaktivieren: dies soll durch die sekretorische Komponente verhindert werden. Das sekretorische IgA kann an Oberflächenrezeptoren auf den Schleimhautepithelien binden, was auch als »antiseptischer Anstrich « bezeichnet wird, und bildet somit auf der Schleimhaut die erste spezifische Front gegen eindringende Erreger (9). Von IgA umhüllte Bakterien werden an ihrer Adhärenz an die Epitheloberfläche gehindert, erste Voraussetzung zur Verhinderung einer Invasion der Bakterien durch das Epithel hindurch.

Lysozym wird nicht nur in den Drüsen der Schleimhaut, sondern auch von neutrophilen Granulozyten und Makrophagen produziert. Diese Substanz ist in fast allen Körperflüssigkeiten vorhanden. Ihr Angriffspunkt sind Glykosidverbindungen in Polysacchariden und Mukopeptiden der Zellwand grampositiver Bakterien. Letztendlich bewirkt Lysozym eine Lyse der Bakterienzellwand .

Interferon wirkt vor allem gegen Viren (Alpha- und Betainterferon), verfügt aber auch über immunodulatorische Eigenschaften (Gammainterferon) (10). Wenige Stunden nach einer Virusinfektion wird Interferon von den virusbefallenen Zellen produziert und danach extrazellulär mit dem Sekret relativ weitflächig verbreitet. Interferon verhindert eine weitere zelluläre Vermehrung der Viren. Gammainterferon wird von T-Zellen und Makrophagen produziert, wirkt als Lymphokin und aktiviert Makrophagen und BZellen, was die Phagozytosetätigkeit z. B. der Makrophagen erhöht. Ferner werden auch natürliche Killerzellen aktiviert, die virusinfizierte Zellen abtöten können.

Das Komplement ist ein weiterer unspezifischer Schutzfaktor. Dieses aus etwa 20 Proteinen bestehende unspezifische Effektorsystem, das kaskadenartig wirkt, hat folgende Hauptfunktionen (Tabelle 2): die Chemotaxis und Aktivierung von Immunzellen,

Tabelle 2. Hauptfunktionen des Komplementsystems.

Chemotaxis und Aktivierung von Immunzellen (z. B. C3a, C5a)

Opsonierung (z. B. C3b)

Bakterienlyse, Zytolyse (C5-9, »MAC«)

z. B. durch die Komplementfaktoren C3a und C5a, sowie ferner die Opsonierung, d. h. die Umhüllung von Bakterien mit Proteinen zur Begünstigung ihrer Aufnahme durch Phagozyten. Akutphaseproteine, z. B. das C-reaktive Protein, sind derartige Opsonine. Ihre Anlagerung an Bakterien bewirkt eine zusätzliche Anlagerung von Komplement, 
z. B. des C3b, über einen C3b-Rezeptor. Die Bindung von Akutphaseprotein und Komplement erleichtert dann die Phagozytose der Bakterien durch Makrophagen. Die dritte wichtige Funktion des Komplementsystems ist die Bakterienlyse und die Zytolyse mittels der Komponenten C5-9 über die Bildung des Membrane-attack-complex (MAC), dessen röhrenartige Struktur durch Zusammenbruch der extraintrazellulären Gradienten der Zellmembran zur Zerstörung der Zelle führt (36).

Weiter Bestandteile des Bronchialsekrets sind Antiproteasen (Proteaseninhibitoren). Bei allen entzündlichen Reaktionen kommt es zum Zellzerfall und damit zur Freisetzung von Proteasen insbesondere auch aus den neutrophilen Granulozyten, welche jedoch Proteasen nicht nur durch Zerfall freisetzen, sondern auch aktiv aus intakten Zellen sezernieren. Weitere Quellen für Proteasen sind die Makrophagen. In der Schleimhaut existiert ein spezifischer Schleimhautinhibitor, der etwa $80 \%$ der Antiproteaseaktivität des Bronchialsekrets ausmacht, neben dem bekannteren Alpha-1-Antitrypsin (39).

\section{Alveoläres Kompartiment}

Der Surfactant wird von dem voluminösen Typ-2-Alveozyten gebildet, der normalerweise nur $7 \%$ der Alveolaroberfläche bedeckt, während 93 \% von den abgeflachten Typ-1Alveozyten eingenommen werden (14). Die Surfactantphospholipide haben die Hauptaufgabe, die Oberflächenspannung der Alveozyten herabzusetzen und einer Atelektasenbildung vorzubeugen (45). Neben dieser Hauptaufgabe wurden in letzter Zeit jedoch auch immunoligische Eigenschaften entdeckt. Kultiviert man menschliche Alveolarmakrophagen in Gegenwart von Surfactantphospholipiden, so wird die zytotoxische Wirkung der Alveolarmakrophagen auf Tumorzellen deutlich verstärkt (4). Ebenso wird im Tiermodell die abtötende Wirkung der Alveolarmakrophagen auf Bakterien durch Surfactant verstärkt und die Phagozytoseaktivität gesteigert (29). Die antigen- oder mitogeninduzierte Lymphozytenproliferation hingegen wird durch Surfactant abgeschwächt (2). Er steigert auch die Migration chemotaktisch stimulierter Alveolarmakrophagen (20). Die genauere Funktion dieser immunologischen Eigenschaften bedarf sicher noch einer weiteren Untersuchung.

Die Alveolarmakrophagen, Zellen der monozytären Reihe, nehmen eine zentrale Stelle im unspezifischen Abwehrsystem ein. Es gibt kaum eine immunologische Reaktion, an der sie nicht direkt oder indirekt (über Monokine, Enzyme oder Entzündungsmediatoren) beteiligt sind. In der gesunden Lunge machen die Alveolarmakrophagen über $85 \%$ der Abwehrzellen in den Alveden aus, wie Untersuchungen der bronchoalveolären Lavage zeigen konnten (6). Eine der Hauptfunktionen gerade des Alveolarmakrophagen ist die Phagozytose inhalierter Staubpartikel. Weitere Eigenschaften sind in Tabelle 3 aufgeführt (11). Er kann, wie bereits erwähnt, auf Mikroorganismen und Tumorzellen zytotoxisch wirken. Diese Prozesse werden über die Produktion toxischer Sauerstoffmetaboliten, z. B. Sauerstoffradikale oder Wasserstoffsuperoxid, oder auch den Tumornekrosefaktor (TNF), der von aktivierten Makrophagen freigesetzt wird (42), vermittelt. Die im Makrophagen enthaltenen Enzyme können eine Gewebeschädigung verursachen, indem sie Elastin und Kollagen abbauen, was im Rahmen der Säuberung entzündlich veränderter Gewebe eine sinnvolle Funktion darstellen kann. Die Gewebereparatur wird durch die Sekretion verschiedener Wachstumsfaktoren für Fibroblasten in Gang gesetzt, die ebenfalls dem Alveolarmakrophagen entstammen: Genannt seien Fibronektin, »Alveolar macrophage derived growth factor« (AMDGF) und »Platelet 
Tabelle 3. Funktionen des Alveolarmakrophagen.

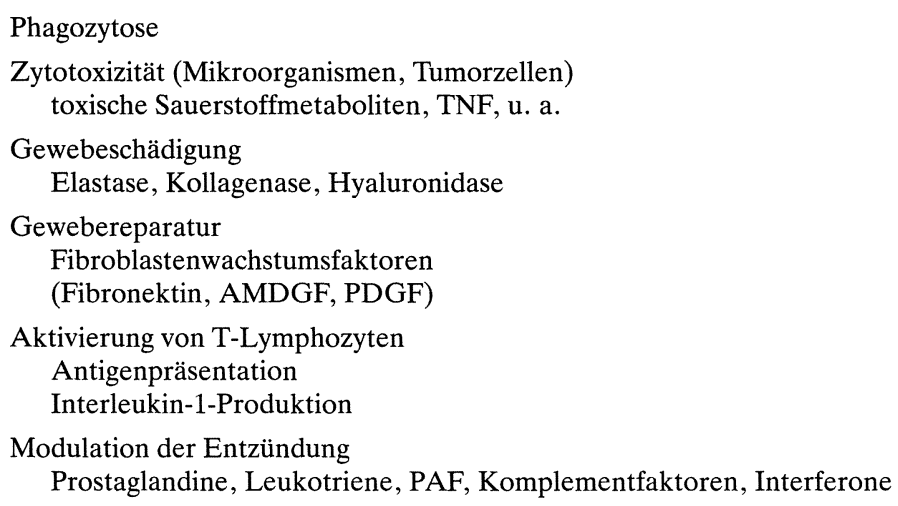

derived growth factor « (PDGF). Ferner erfüllt der Alveolarmakrophage eine wichtige Funktion bei der Aktivierung von T-Lymphozyten, indem er Antigen präsentiert und Interleukin-1 bildet. Auf die Modulation der Entzündung durch die Freisetzung von Mediatoren wie Prostaglandine, Leukotriene, Platelet activating factor (PAF), Komplementfaktoren und Interferone auch aus Makrophagen sei ebenfalls hingewiesen (25).

\section{Immunologische Abwehr}

Die immunologische Abwehr läßt sich in ein natürliches (unspezifisches) und adaptives (spezifisches) Abwehrsystem einteilen (Tabelle 4). Beide ergänzen sich in ihrer Wirkung. Das entwicklungsgeschichtlich ältere natürliche System verfügt über ein wenig differenziertes Erkennungsvermögen ohne »Gedächtnis«, ist jedoch mit hoher phagozytischer und zytotoxischer Aktivität ausgestattet. Das jüngere, nur bei Vertebraten vorkommende adaptive Immunsystem weist ein sehr spezifisches Erkennungsvermögen auf, welches eine antigenspezifische klonale Reaktivität der T- und B-Lymphozyten induziert und zur

Tabelle 4. Lösliche und zelluläre Komponenten des immunologischen Abwehrsystems.

\begin{tabular}{lll}
\hline & $\begin{array}{l}\text { Natürliche Immunität } \\
\text { unspezifisch }\end{array}$ & $\begin{array}{l}\text { Adaptive Immunität } \\
\text { spezifisch }\end{array}$ \\
\hline lösliche & $\begin{array}{l}\text { Lysozym } \\
\text { Komponenten } \\
\text { Akute-Phase-Proteine } \\
\text { Interferone } \\
\text { Mollen }\end{array}$ & Antikörper \\
& $\begin{array}{l}\text { Monozyten/Makrophagen } \\
\text { Granulozyten } \\
\text { NK-Zellen }\end{array}$ & T- und B-Lymphozyten \\
\hline
\end{tabular}


Ausbildung kurzfristig reaktivierbarer Gedächtniszellen führt, so daß die antigenspezifischen Reaktionen der Immunzellen mit jedem neuen Antigenkontakt intensiver ausfallen.

Die Funktion des Makrophagen wurde bereits dargestellt. Im folgenden soll auf weitere Immunzellen eingegangen werden, die ebenfalls in den Alveolen vorkommen und bei zahlreichen Erkrankungen in der brochoalveolären Lavage vermehrt nachgewiesen werden können.

Neutrophile Granulozyten. In der entzündungsfreien Lunge finden sich intraalveolär nur spärlich neutrophile Granulozyten (etwa 1 pro 100 Alveolen). Die Lungenkapillaren hingegen dienen als Reservoir für rasch verfügbare Neutrophile. Physiologisch kommt den Neutrophilen eine Rolle in der unspezifischen Abwehr von bakteriellen und von Pilzinfektionen zu. Sie werden durch chemotaktische Faktoren (C5a, Leukotrien B4, 5HETE, Interleukin-1) sowie durch Phagozytose (überwiegend Fc-rezeptor- und C3brezeptor-vermittelt) opsonierter Krankheitserreger aktiviert. Nach Aktivierung produzieren sie große Mengen an Oxidantien (toxische Sauerstoffradikale wie $\mathrm{O}_{2}{ }^{-}, \mathrm{OH}^{-}$), wodurch die Krankheitserreger effektiv abgetötet werden (30). Eine zweite Eigenschaft ist die Freisetzung proteolytischer Enzyme wie Elastase, Kollagenase und Kathepsin G, die dem Abbau von Zelldetritus im Entzündungsgebiet dienen.

Diese in der Beherrschung von Infektionenkrankheiten äußerst sinnvollen Mechanismen kommen jedoch auch bei einer Reihe von nicht-infektiösen Krankheiten zum Tragen, in deren Pathogenese der Neutrophile durch toxische Sauerstoffradikale und proteolytische Enzyme zur Zellschädigung und Zerstörung der extrazellulären Bindegewebsmatrix beiträgt (38). In der Pneumologie sind Beispiele hierfür die Schocklunge (ARDS); (41), die Neutrophilenalveolitis bei idiopathischer Lungenfibrose (16), die akute Phase der exogen allergischen Alveolitis (7) sowie die chronisch-obstruktive Bronchitis mit Lungenemphysem (39).

Eosinophile Granulozyten. Bis vor wenigen Jahren schrieb man dem eosinophilen Granulozyten ausschließlich eine Rolle bei der Abwehr von Parasiten und bei der Modulation inflammatorischer Reaktionen zu (37). In der Zwischenzeit stellte sich jedoch mehr und mehr heraus, daß der Eosinophile im Rahmen entzündlicher Reaktionen nicht nur eine modulatorische Funktion, sondern eine Position als zentrale Effektorzelle einnimmt. Der Eosinophile stammt aus dem Knochenmark und reift unter T-lymphozytärer Kontrolle zur aktivierten Effektorzelle heran (23). Er besitzt einen bilobulären Nukleus, ein ausgeprägtes endoplasmatisches Retikulum, einen aktiven Golgi-Apparat sowie zwei verschiedene Typen von Granula. Die sogenannten »spezifischen Granula « sind durch eine elektronendichte, kristalloide Matrix gekennzeichnet und enthalten die eosinophile Peroxidase (EPO), ß-Glucuronidase, Histaminase und die drei charakteristischen eosinophilen basischen Proteine Major basic protein (MBP), Eosinophil cationic protein $(E C P)$ sowie Eosinophil-derived neurotoxin (EDN). Die sogenannten »kleinen Granula « treten vor allem in reifen Eosinophilen auf und enthalten Arylsulfatase und saure Phosphatase. Schließlich sind einige funktionell wichtige Enzyme (Ysophosphatase oder Charcot-Leyden-Kristall, Phosphopholipase $\mathrm{A}_{2}$ und D) an der Membran lokalisiert (15, 28, 32, 37, 40). Tabelle 5 gibt eine Übersicht über die Funktion dieser Mediatoren und Enzyme.

Die proinflammatorischen und zytotoxischen Wirkungen des Eosinophilen werden durch vier Effektormechanismen vermittelt:

1. eosinophile Enzyme (Peroxidase, Kollagenase, Lysozym); 


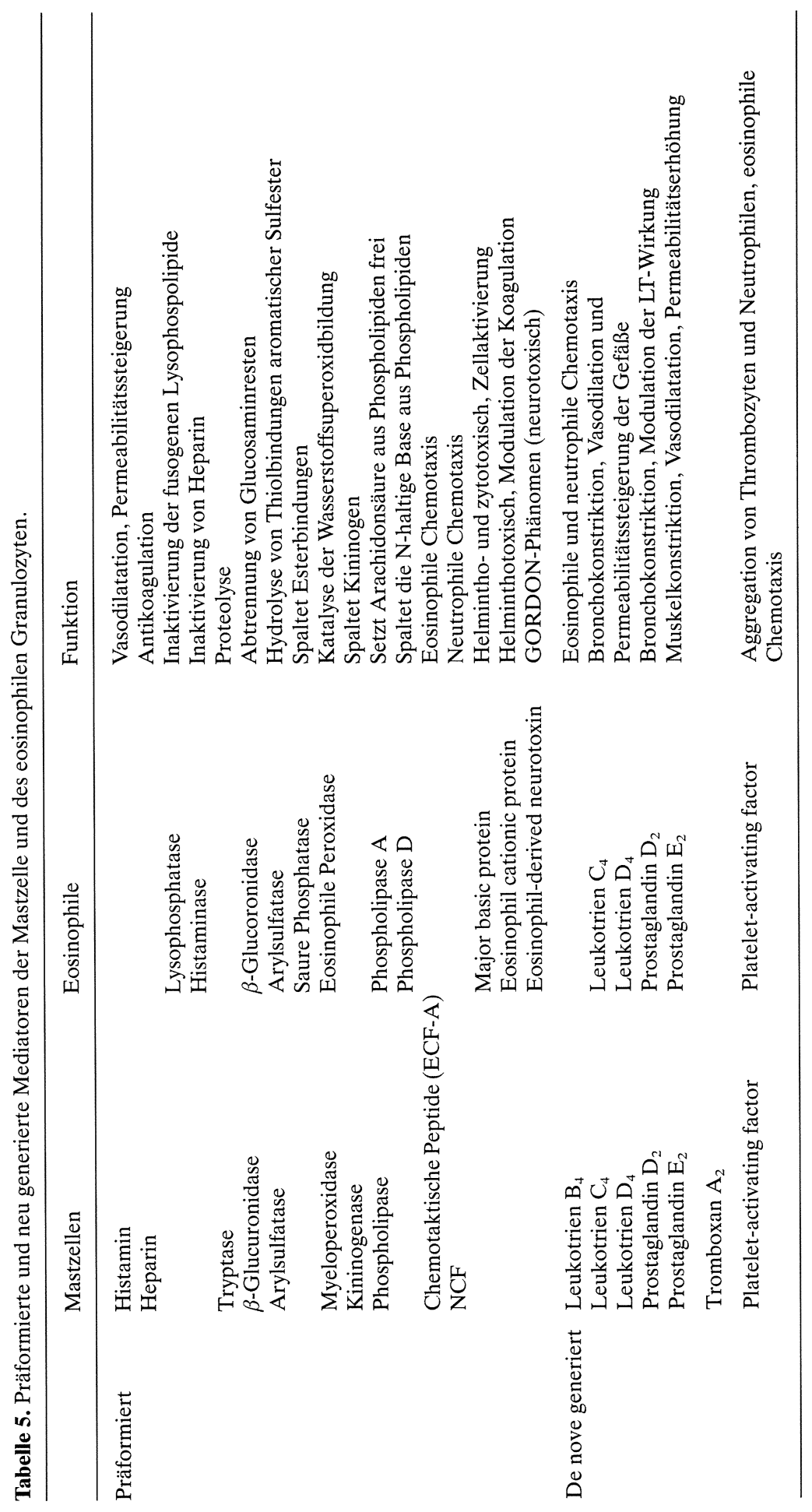


2. de novo generierte und präformierte Mediatoren (Leukotriene, Platelet-activating factor (PAF), Prostaglandine, Histamin);

3. Sauerstoffmetaboliten $\left({ }^{1} \mathrm{O}_{2}, \mathrm{O}_{2}{ }^{-}, \mathrm{OH}^{-}\right)$;

4. eosinophile basische Proteine (MBP, ECP, EDN).

Eosinophile sind schlechtere Phagozyten als Neutrophile, nehmen jedoch AntigenAntikörperkomplexe besser auf und phagozytieren auch Mastzellgranula (8).

Physiologisch kommt den eosinophilen Granuluzyten eine wichtige Rolle bei der Abwehr parasitärer Infektionen zu (44). Zunächst stimulieren parasitäre Antigene TLymphozyten sowie Makrophagen, die ihrerseits B-Lymphozyten zur Synthese spezifischer Antikörper veranlassen (IgG, IgE). Unter dem Einfluß mastzellabhängiger (PAF, $\mathrm{LTB}_{4}$, eosinophile Peptide) und parasitärer chemotaktischer Faktoren (ECFp) kommt es zur lokalen Ansammlung eosinophiler Granulozyten. Über spezifische IgE- und IgGAntikörper wird der Eosinophile direkt an den Helminthen gebunden. Danach kommt es zur Freisetzung eosinophiler Enzyme, basischer Proteine sowie toxischer Sauerstoffmetaboliten, die gemeinsam den Parasiten abtöten. Das komplexe Zusammenwirken verschiedener Zellen bei der Abwehr von Parasiten ist in Abb. 1 dargestellt.

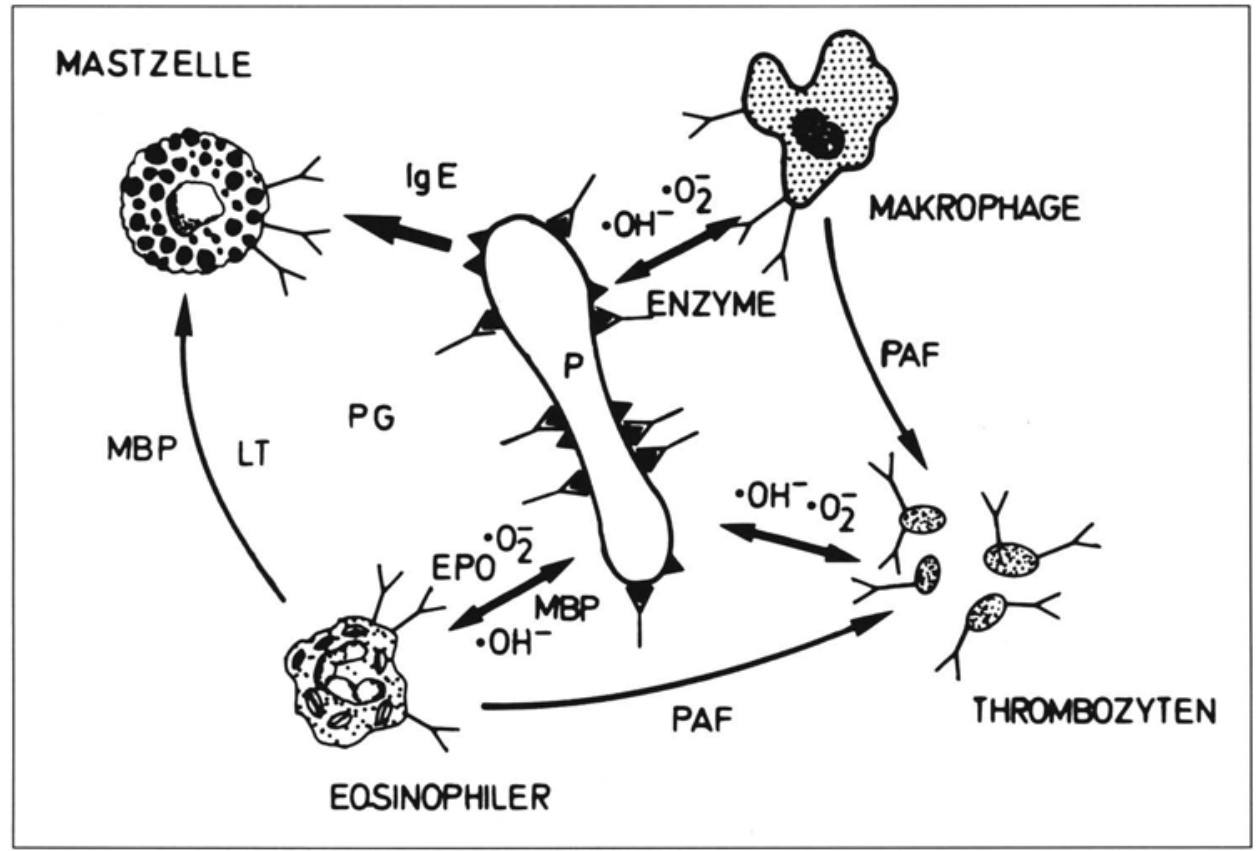

Abb. 1. Kooperation von Eosinophilen, Mastzellen, Makrophagen und Thrombozyten bei der Abwehr von Parasiten. (EPO eosinophile Peroxidase; LT Leukotriene; MBP Major basic protein; PAF Plateletactivating factor; PG Prostaglandine)

In der Pathophysiologie spielen Eosinophile bei verschiedenen Erkrankungen ebenfalls eine wichtige Rolle. Bekannt geworden sind Eosinophilenvermehrungen im peri- 
pheren Blut außer bei Patienten mit Parasitenbefall auch beim Asthma bronchiale (sowohl bei exogen-allergischem als auch bei Intrinsic Asthma), beim hypereosinophilen Syndrom, bei bestimmten Vaskulitiden bzw. Granulomatosen, bei Kollagenosen sowie bei Malignomen. Auch in der bronchoalveolären Lavage treten Eosinophile als Ausdruck der Lungengewebsinfiltration bei diesen Krankheiten vermehrt auf, ebenso bei der idiopathischen Lungenfibrose, bei der jedoch eine Bluteosinophilie nicht zu beobachten ist (16).

Die Wirkung der eosinophilen Effektormechanismen läßt sich am Beispiel des Asthma bronchiale verdeutlichen. Ein vergleichbarer Effekt muß jedoch grundsätzlich auch bei anderen hypereosinophilen Erkrankungen angenommen werden. Die Akkumulation der Eosinophilen erfolgt sowohl durch IgE-abhängige als auch IgE-unabhängige Reaktionen mittels verschiedener chemotaktischer Faktoren. In der Bronchialwand bzw. dem Bronchiallumen kommt es unter dem Einfluß anderer Entzündungszellen zur Freisetzung eosinophiler Enzyme, basischer Proteine und toxischer Sauerstoffmetaboliten (10, 28, 40). So läßt sich beispielsweise MBP in allen Bereichen der geschädigten Asthmalunge sowie im Sputum von Asthmatikern in großen Mengen nachweisen (13). Das MBP ist vermutlich wesentlich für die charakteristische Zerstörung des mukoziliären Apparates verantwortlich (17). Die ebenfalls MBP-mediierte Destruktion der bronchialen Epithelbarriere oder die Aktivierung von Mastzellen und basophilen Granulozyten (27) sowie die durch Eosinophile induzierte kapilläre Vasodilatation (46) dürfte im Sinne eines Circulus vitiosus zu einer verstärkten Akkumulation eosinophiler Granulozyten, aber auch anderer Entzündungszellen führen. Auf diese Weise wird die Freisetzung von Entzündungsmediatoren (Leukotrien $\mathrm{C}_{4}$, Leukotrien $\mathrm{D}_{4}, \mathrm{PAF}$, Prostaglandine) unterhalten. Dies führt schließlich zur weiteren Gewebsalteration und Bronchokonstriktion (22). Neben dem MBP vermag auch das ECP über die Ausbildung transmembranöser Poren Bronchialzellen zu schädigen (47). Zusätzlich bewirkt eine intragranulär gespeicherte eosinophile Kollagenase die Zerstörung der kollagenhaltigen Lamina propria (3).

Mastzellen. Sie spielen eine zentrale Rolle im Rahmen allergischer Reaktionen. In den meisten Fällen werden sie durch IgE-abhängige Immunreaktionen getriggert. Darüberhinaus können aber auch natürlich vorkommende Peptide, wie Melittin oder das Mast cell degranulating peptide (MCD), sowie Medikamente wie Polymyxin die Zelle direkt aktivieren (26). Die Aktivierung führt zur Freisetzung präformierter und neu generierter Mediatoren (siehe Tabelle 5), die in vielfacher Weise die lokale Entzündungsreaktion beeinflussen. Ferner ist die Mastzelle bei einer Reihe anderer pathophysiologischer Prozesse beteiligt, wie der Abwehr von Parasiten durch die eosinophilen Granulozyten (siehe Abb. 1) oder der Zerstörung von Tumorzellen (18). Zwischen Mastzellen und T-Lymphozyten besteht ein wechselseitiger Kontakt. Einerseits führen lymphozytäre Faktoren zur Mastzellaktivierung und Mastzelldegranulation (5). Andererseits vermag Histamin T-Suppressorlymphozyten zu aktivieren, die wiederum der Bildung von Granulomen entgegenwirken (44). Histamin beeinflußt auch die Aktivität von Bindegewebsfibroblasten (5) und ist auf diese Weise an der Pathogenese chronischer Entzündungen bis hin zur Fibrose beteiligt. Schließlich wirken die neu generierten Mediatoren wie Prostaglandine und Leukotriene auch auf verschiedene Funktionen der lymphozytären Immunreaktion (33).

Lymphozyten. In Tabelle 6 ist schematisch der Dualismus des T-und B-Zellsystems dargestellt. Beiden Zellsystemen ist gemeinsam, daß sie Rezeptorstrukturen für die Erkennung des homologen Antigens aufweisen. Ein prinzipieller Unterschied zwischen 
Tabelle 6. Der Dualismus des T- und B-Zellsystems.

\begin{tabular}{|c|c|}
\hline T-Lymphozyt & B-Lymphozyt \\
\hline Zelluläre Immunität & Humorale Immunität \\
\hline Erkennung zellgebundener Antigene & Erkennung frei zirkulierender Antigene \\
\hline $\begin{array}{l}\text { Zytotoxische Effektorzellen } \\
\quad \text { (Viren, Pilze, Tumorzellen, Transplantate) }\end{array}$ & Differenzierung zu Plasmazellen \\
\hline $\begin{array}{l}\text { Immunregulation } \\
\text { (Hilfe/Suppression) }\end{array}$ & $\begin{array}{l}\text { Antikörperproduktion } \\
\text { (Bakterien, Parasit) }\end{array}$ \\
\hline \multicolumn{2}{|l|}{ Lymphokinproduktion } \\
\hline Typ-IV-Reaktion & Typ-I-, Typ-II-, Typ-III-Reaktion \\
\hline
\end{tabular}

beiden besteht jedoch darin, daß T-Lymphozyten nur zellgebundene Antigene erkennen, während B-Lymphozyten frei zirkulierende Antigene erkennen und binden können. Während der B-Lymphozyt zu Plasmazellen differenziert, welche dann die spezifische Antikörperproduktion vornehmen, was hauptsächlich gegen bakterielle und parasitäter Infekte gerichtet ist, und an pathologischen Immunreaktionen die allergischen Reaktionen vom Typ I, Typ II und Typ III vermittelt, hat der T-Lymphozyt die Funktion der zytotoxischen Effektorzelle (T8-positiv), das heißt er kann virusinfizierte Zellen, Tumorzellen oder Transplantatzellen direkt angehen und vernichten, ohne daß dies über lösliche Antikörper vermittelt wird. Weiterhin haben T-Zellen die wichtige Aufgabe der Immunregulation. Sie können die B-Zellreaktion fördern oder supprimieren. Begegnet der B-Lymphozyt einem spezifischen Antigen, kann das die Umwandlung in eine antikörpersezernierende Plasmazelle auslösen. Die Umwandlung selbst wird durch verschiedene Unterklassen von T-Lymphozyten reguliert, die entweder als Helfer, das sind die T4-positiven T-Lymphozyten, oder als Unterdrücker bzw. Suppressor arbeiten; letzteres sind T8-positive T-Lymphozyten. Über Lymphokinbildung mit Aktivierung der Makrophagen spielen die T-Zellen ferner eine wichtige Rolle als T4-positive Effektorzellen der allergischen Typ- IV-Reaktion, die zum Beispiel bei der Tuberkulose, bei Mykosen und auch bei der Sarkoidose zum Tragen kommt. Dabei sind die wichtigsten Lymphokine die Interleukine, Interferone, monozyten-chemotaktische Faktoren, migrations-inhibitierende Faktoren, makrophagen-aktivierende Faktoren sowie der Tumornekrosefaktor (10).

Antigenpräsentation und T-Zellaktivierung. Der T-Lymphozyt erkennt Antigen nur, wenn es ihm von einem Makrophagen präsentiert wird. Antigen-präsentierende Zellen stellen eine spezialisierte Form von Gewebsmakrophagen dar. Sie finden sich unter anderem auch in der Lunge (43). Diese antigen-präsentierenden Zellen nehmen das Antigen, zum Beispiel einen Viruspartikel, auf und bieten es an ihrer Zelloberfläche einem spezifischen T-Lymphozyten dar. Der T-Lymphozyt kann das Antigen auf dem Makrophagen jedoch nur in Verbindung mit einem MHC-Protein (HLA-Antigen), welches auf der Oberfläche des Makrophagen exprimiert wird, erkennen. Auch im Modus der Antigenerkennung unterscheiden sich T4- und T8-Zellen voneinander. Die T4-Zelle erkennt das Antigen in Verbindung mit MHC Klasse II, während die T8-Zelle das Antigen in Verbindung mit MHC Klasse I erkennt (34). 


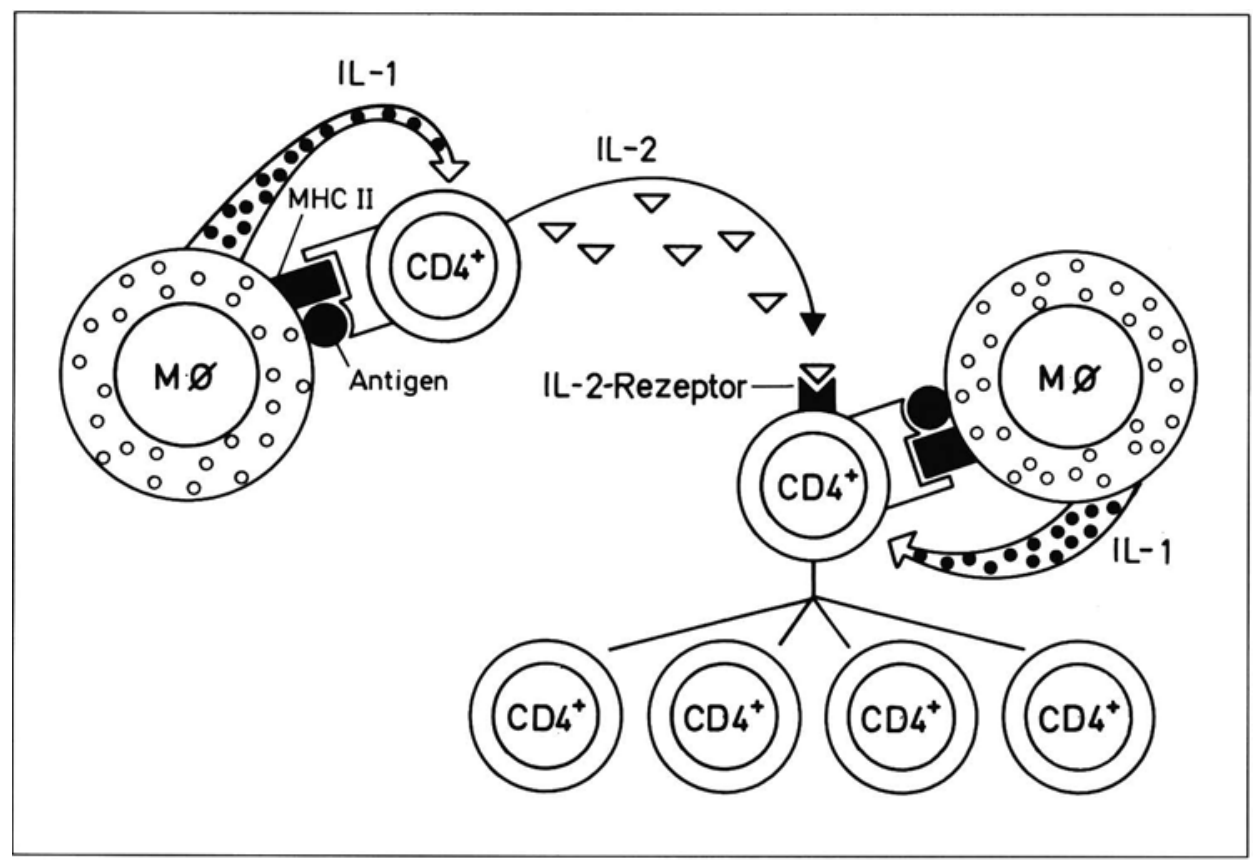

Abb. 2. Mechanismus der klonalen T-Zellproliferation.

Wenn der T4-Zelle nun das passende Antigen in Verbindung mit MHC-II präsentiert wird und zusätzlich noch Interleukin-1 vom Makrophagen gebildet wird, ist die T4-Zelle aktiviert (Abb. 2). Die aktivierten T-Zellen produzieren nun selbst wiederum Botenstoffe, die als Lymphokine bezeichnet werden. Eine solche Substanz ist das Interleukin2 (12). Während eine Population von aktivierten T4-Zellen Interleukin-2 bildet, exprimieren andere, gleichermaßen aktivierte T4-Zellen den Interleukin-2-Rezeptor. Trifft nun das Interleukin-2 auf seinen Rezeptor, wird die klonale Expansion aktivierter, antigenspezifischer T-Lymphozyten in Gang gesetzt (Abb. 2).

Bei einer Virusinfektion zum Beispiel ist das Ziel die Bereitstellung von genügend spezifischen zytotoxischen T-Zellen, die in der Lage sind, virusinfizierte Zellen abzutöten. Mit der Vernichtung der befallenen Zellen kann in diesem Stadium die Infektion schnell gestoppt werden. Etwas langsamer als die Zahl der zytotoxischen Zellen wächst die der Suppressorzellen, die ebenfalls T8-positiv sind. Sie tragen dazu bei, die TZellantwort abzuschalten. Bei dieser T-Zellantwort spielen T4-Helfer und T4-Inducerzellen die zentrale Rolle, ohne deren Einwirkung, sei es durch Lymphokine oder durch direkten Kontakt, weder die zytotoxischen noch die Suppressorzellen ihre Aufgabe erfüllen können. Weiterhin beeinflussen die T4-Zellen durch das von ihnen abgegebene Interleukin-2 auch die natürlichen Killerzellen, die antigenunabhängig virusinfizierte Zellen oder Tumorzellen abtöten können.

In Abb. 3 ist die zentrale Rolle der T4-Zelle bei der Immunantwort dargestellt. Zur optimalen B-Zellantwort (links) ist die Mithilfe der T4-positiven Helferzellen eine obli- 
gate Voraussetzung. Gerade diese T4-Helferzelle geht jedoch bei der HIV-Infektion zugrunde (19). In der Mitte der Abbildung ist die T-Zellreaktion auf das Antigen zu sehen. Das Antigen wird vom Makrophagen in Verbindung mit MHC Klasse II präsentiert. Unter Mitwirkung der T4-positiven Zelle entstehen danach die Suppressorzellen und auch die zytotoxischen Zellen. Die zytotoxischen Zellen können direkt eine virusinfizierte Zelle, die Virusantigen auf der Membran exprimiert, zerstören (rechts in Abb.3).

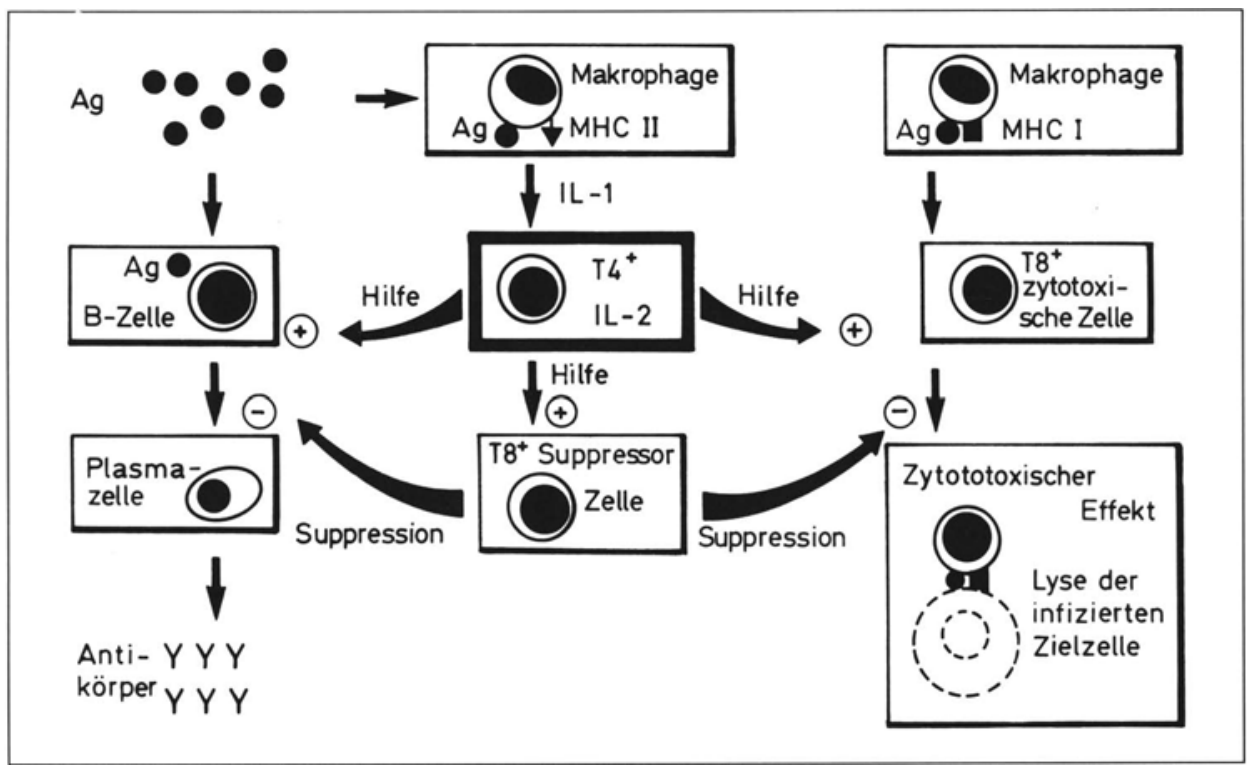

Abb. 3. Zentrale Rolle der T4-Zellen: Helferfunktion für die B-Zellantwort und die zytotoxische T-Zellreaktion.

Bei HIV-Infektion ist einmal die Zahl der T4-positiven Zellen reduziert. Die Konsequenzen der ausbleibenden Helferfunktion sind, daß die B-Zellen nicht mehr ausreichende Mengen spezifischer Antikörper gegen das AIDS-Virus oder auch andere Erreger produzieren. Ähnlich beeinträchtigt ist auch die Reaktion der zytotoxischen Zellen. Die T-Suppressorzellen können ebenfalls ihre Aufgaben nicht mehr erfüllen, was zum Beispiel die unaufhörlich große Menge unspezifischer Immunglobuline erklären könnte, die von den B-Zellen bei Patienten mit AIDS produziert werden. Weniger Interleukin-2 und weniger Gammainterferon bedeuten zugleich auch eine geringere Aktivität der natürlichen Killerzellen und der Makrophagen (19).

Kürzlich wurde eine weitere, verheerende Entdeckung publiziert. Eine französische Arbeitsgruppe stellte fest, daß nicht nur Lymphozyten, sondern auch bis zu 60 Prozent der Alveolarmakrophagen bei HIV-positiven Patienten mit dem HI-Virus infiziert sind (35). Auch Makrophagen exprimieren nämlich auf ihrer Oberfläche zum Teil das T4Antigen, das als Rezeptor für die Bindung des HI-Virus an die Zellmembran und seine Einschleusung in die Zielzelle dient. Gleichzeitig konnten aus der Lavageflüssigkeit von HIV-positiven Patienten HIV-spezifische zytotoxische T-Lymphozyten isoliert werden. 
Diese alveolären zytotoxischen T-Zellen sind in der Lage, die HIV-befallenen Alveolarmakrophagen zu vernichten. Die Konsequenz dieser Beobachtungen ist klar: Durch die Zerstörung HIV-infizierter eigener Alveolarmakrophagen wird auch dieser Teil der unspezifischen Immunantwort erheblich beinträchtigt. Die Immunabwehr bricht in den Alveolen bereits an der ersten Frontlinie der spezialisierten Phagozyten zusammen, was die Invasion der Lungen mit den verschiedensten, zum Teil exotisch anmutenden Erregern zur Folge hat, und auch das überaus häufige Auftreten von Pneumocystis-cariniiPneumonien bei AIDS, verglichen mit anderen immungeschwächten Patienten, zu erklären vermag.

\section{Literatur}

1. Albegger K (1980)Nicht-immunologische Abwehrmechanismen des oberen Resporationstraktes. In: Intorp HW, Nolte D (Hrsg) Immunabwehr des Repirationstrakts. Dustri, München-Deisenhofen, S $37-46$

2. Ansfield MJ, Benson BJ (1980) Idenfication of the immunosuppressive components of canine pulmonary surface-active material. J Immunol 125:1093-1098

3. Arars GH, Altman LC, Gleich GJ, Loeggering DA (1985) Eosinophil- and eosinophil granulemediated pneumocyte injury. J Allergy Clin Immunol 76:595-604

4. Baughman RP, Mangels D, Corser BC (1987) Phospholipids in surfactant enhanced monocyte/ macrophage tumor cytotoxity. Chest 91:28S-29S

5. Bienenstock J, Tomioka M, Stead R, Ernst P, Jordana M, Gauldie J, Dolovich J (1987) Mast cell involvement in various inflammatory processes. Am Rev Respir Dis 125:S5-8

6. Costabel U, Bross KJ, Matthys H (1985) Bronchoalveoläre Lavage: Klinische Bedeutung zytologischer und immunzytologischer Befunde. Prax Klin Pneumol 39:343-355

7. Costabel U (1988) The alveolitis of hypersensitivity pneumonitis. Eur Respir J 1:5-9

8. Czarnetzki BM (1987) Mastzellen, Eosinophile, Makrophagen. In: Fuchs E, Schulz KH (Hrsg) Manuale allergologicum. Dustri, München-Deisenhofen, S III.2:1-10

9. Deicher H (1987) Das Immunsystem.In: Fuchs E, Schulz KH (Hrsg) Manuale allergologicum. Dustri, München-Deisenhofen, S III.1:1-29

10. Dinarello CA, Mier JW (1987) Current concepts: lymphokines. N Engl J Med 317:940-945

11. Fels AOS, Cohn ZA (1986) The alveolar macrophage. J Appl Physiol 60(2):353-369

12. Fink R, Dancygier H (1987) Interleukin-2. Dtsch med Wschr 112:188-193

13. Frigas E, Loeggering DA, Solley GO, Farrow GM (1981) Elevated levels of eosinophil granule major basic protein in the sputum of patients with brochial asthma. Mayo Clin Proc 56:345-53

14. Gehr P (1987) Anatomische Grundlagen für den Gasaustausch in der Lunge. Atemw Lungenkrkh 13:217-223

15. Gleich GJ, Loeggering DA (1984) Immunbiology of eosinophils. Ann Rev Immunol 2:429-59

16. Haslam PL, Turton CWG, Lukoszek A, Collins JV, Salesbury AJ Turner-Warwick M (1980) Bronchoalveolar lavage fluid cell counts in cryptogenic fibrosing alveolitis and their relation to therapy. Thorax 35:328-339

17. Hastie AT, Loeggering DA, Gleich GJ, Kueppers F (1987) The effect of purified eosinophil major basic protein on mammalian ciliary activity. Am Rev Respir Dis 135:848-53

18. Henderson WR, Chi EY, Jong EC, Klebanoff SJ (1981) Mast cell-mediated tumor-cell cytotoxity. Role of the peroxidase system. J Exp Med 153:520-33

19. Ho DD, Pomerantz, Kaplan JC (1987) Pathogenesis of infection with human immunodeficiency virus. N Engl J Med 317:278-286

20. Hoffmann RM, Claypool WD, Katyal SL, Singh G, Rogers RM, Dauber JH (1987) Augmentation of rat alveolar macrophage migration by surfactant protein. Am Rev Respir Dis 135:1358-1362

21. Intorp HW (1980) Funktion und biologische Bedeutung des lokalen Immunsystems. In: Intorp HW, Nolte D (Hrsg) Immunabwehr des Respirationstrakts. Dustri, München-Deisenhofen, S 17-36

22. Kauffman HF, Van der Belt B, DeMonchey JGR, Boelens H (1987) Leukotrien C 4 production by normal-density and low-density eosinophils of atopic individuals and other patients with eosinophilia. J Allergy Clin Immunol 79:611-19 
23. Kay AB (1987) Eosinophils in immunological reactions. Clin Allergy 17:251-58

24. Konietzko N, Nakhosteen JA, Mizera W, Kasparek R, Hesse H (1981) Ciliary beat frequency of biopsy samples taken from normal persons and persons with various lung diseases. Chest 80:855857

25. König W,Peiffer P, Schönfeld W, Knöller J (1987) Immunpathologie des oberen Respirationstrakts. Arch Oto-Rhino-Laryngol (Suppl I):1-84

26. Kroegel C, König W, Mollay C, Kreil G (1981) Generation of the eosinophil chemotactic factor (ECF) from polymorphonuclear neutrophils, rat peritoneal mast cells and mast cell-depleted peritoneal cells by melittin. Mol Immunol 18:227-36

27. Kroegel C, Costabel U, Matthys H (1987) Mechanism of membrane damage mediated by eosinophil major basic protein. Lancet I:1380-81

28. Kroegel C, Costabel U, Matthys H. Die pathogenetische Bedeutung des eosinophilen Granulozyten. Dtsch Med Wschr (Manuskript eingereicht)

29. LaForce FM (1976) Effect of alveolar lining material on phagocytic and bactericial activity of lung macrophages against Staphylococcus aureus. J Lab Clin Med 88:691-99

30. Malech HL, Gallin JI (1987) Current concepts: Immunology. Neutrophils in human diseases. N Engl J Med 317:687-694

31. Nossal GJV (1987) Current concepts: Immunology. The basic components of the immune system. N Engl J Med 316:1320-1325

32. Parmley RT, Spicer SS (1974) Cytochemical and ultrastructural identification of a small type granula in human late eosinophils. Lab Invest 30:557-67

33. Payan DG, Goetzl EJ (1984) Recognition of leukotrien $B_{4}$ by a unique subset of human T-lymphocytes. J Allergy Clin Immunol 74:403-6

34. Peter HH, Pichler WJ (1984) Prinzipien der spezifischen Immunantwort und ihrer Störungen. Fortschr Antimikr Antineoplast Chemother 3-6:825-832

35. Plata F, Autran B. Martins LP, Wain-Hobson S, Raphael M, Mayaud C, Denis M, Guillon JM, Debré P (1987) AIDS virus specific cytotoxic T-lymphocytes in lung disoders. Nature 328:348-351

36. Roitt I, Brostoff J, Male D (1985) Immunology. Gower Medical Publishing, London New York, pp 7.1-7.14

37. Schatz M, Wassermann S, Patterson R (1982) The eosinophil and the lung. Arch Intern Med 142:151519

38. Sibille Y, Lwebuga-Mukasa JS, Polomski, Merrill WW, Ingbar DH, Gee JB (1986) An in vitro model for polymorphonuclear-leukocyte-induced injury to an extra-cellular matrix. Am Rev Respir Dis 134:134-140

39. Snider GL (1984) Two decades of research in emphysema. Schweiz Med Wschr 114:898-906

40. Spry CJF (1985) Synthesis and secretion of eosinophils granule substances. Immunol Today 6:332-5

41. Tate RM, Repine JE (1983) Neutrophils and the adult respiratory distress syndrome. Am Rev Respir Dis 128:552-559

42. Urban JL, Shepard HM, Rothstein JL, Sugarman BJ, Schreiber H (1986) Tumor necrosis factor: a potent effector molecule for tumor cell killing by activated macrophages. Proc Natl Acad Scir USA 83:5233-5237

43. Venet A, Hance AJ, Saltini C, Robinson BWS, Crystal RG (1985) Enhanced alveolar macrophagemediated antigen-induced T-lymphocyte proliferation in sarcoidosis. J Clin Invest 75:293-301

44. Weistock JV, Chensue SW, Boros DL (1983) Modulation of granulomatous hypersensitivity. V. Participation of histamine receptor positive and negative lymphocytes in granulomatous response of Schistomiasis mansoni-infected mice. J Immunol 130:423-7

45. Wright JR, Clements JA (1987) State of art: metabolism and turnover of lung surfactant. Am Rev Respir Dis 135:426-444

46. Yoshida H, Anan S, Hori M, Akiyama T, Ushijima N (1982) Ultrastructural studies of eosinophils in passive cutaneous anaphylaxis. Arch Dermatol Res 272:197-200

47. Young JDE, Peterson CGB, Venge P, Cohn ZA (1986) Mechanism of membrane damage mediated by human eosinophils cationic protein. Nature 321:613

Anschrift des Verfassers:

Priv.-Doz. Dr. med. U. Costabel

Ruhrlandklinik

Tüschener Weg 40

4300 Essen 16 